Supporting Information for:

\title{
Coulomb Barrier for Sequential Two-Electron Transfer in a Nano-Engineered Photocatalyst
}

\author{
Junhui Wang, $₫$ Tao Ding, ${ }^{\ddagger}$ and Kaifeng $\mathrm{Wu}^{*}$ \\ State Key Laboratory of Molecular Reaction Dynamics and Dynamics Research \\ Center for Energy and Environmental Materials, Dalian Institute of Chemical \\ Physics, Chinese Academy of Sciences, Dalian, Liaoning 116023, China \\ * Corresponding Author: kwu@dicp.ac.cn \\ \$. Wang and T. Ding contributed equally to this work.
}

\section{Content list:}

Figures S1-S1 1

Tables S1 and S2

Sample preparations

TA experiment set-ups

Calculation of trion lifetimes

Population analysis in ppp-TA experiments

Kinetics fitting models and electron transfer efficiency

Dissociation-limited long-range electron transfer model

Trion binding energy 

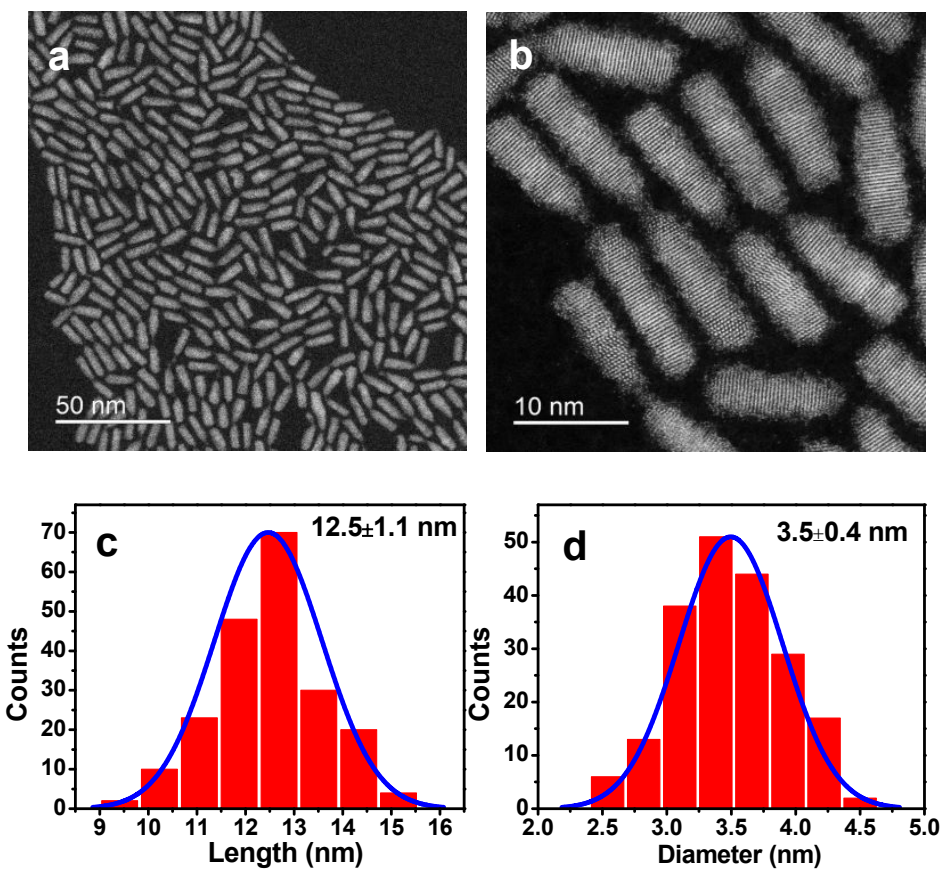

Figure S1. (a) A typical high-angle annular dark-field scanning transmission electron microscopy (HAADF-STEM) image and (b) a high resolution HAADF-STEM image of the CdSe@CdS DIRs used for the size statistics. (c) A statistics distribution diagram for DIR length from which an average length (with a standard deviation) of $12.5 \pm 1.1 \mathrm{~nm}$ is obtained. (d) A statistics distribution diagram for DIR diameter, from which an average diameter of $3.5 \pm 0.4 \mathrm{~nm}$ is obtained.
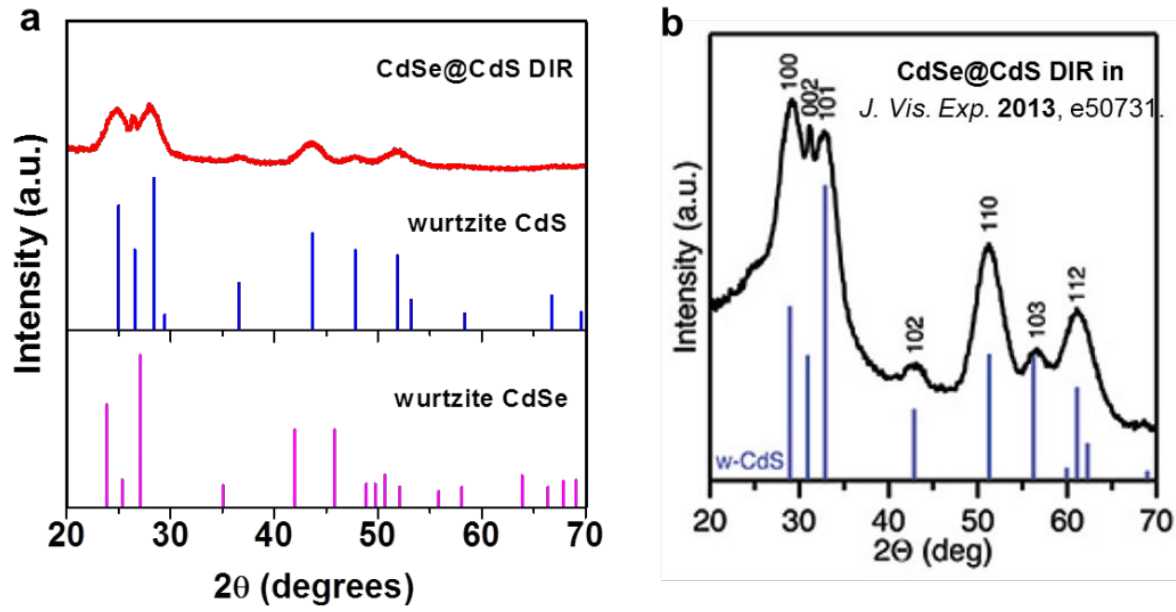

Figure S2. (a) XRD pattern of the CdSe@CdS DIRs in comparison to the standards for bulk wurtzite CdS and CdSe. (b) XRD pattern of the CdSe@CdS DIRs reported in J. Vis. Exp. 2013, e50731. 


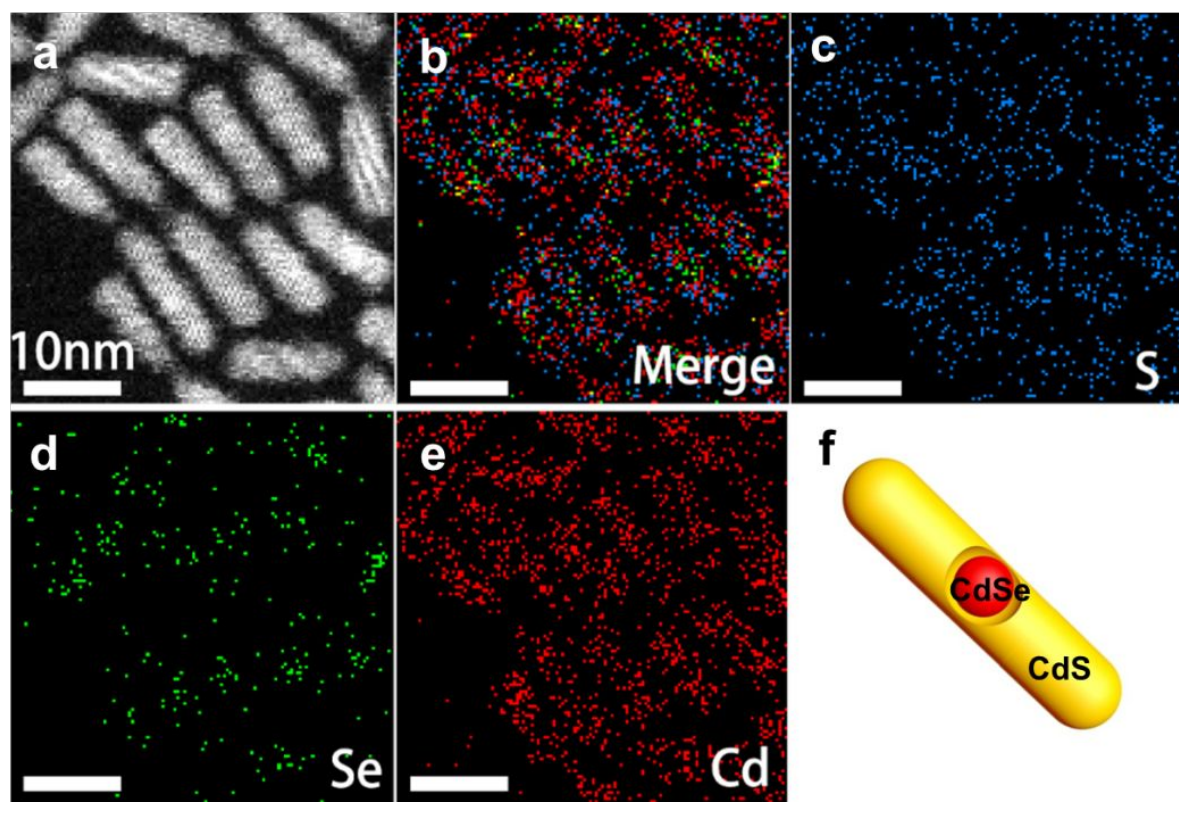

Figure S3. (a) A HAADF-STEM image of the DIRs for elemental mapping. (b) Overlaid energy-dispersive X-ray (EDX) maps of S (blue), Se (green) and Cd (red). (c-e) Individual EDX maps of S (blue, c), Se (green, d) and Cd (red, e). Based on the overlaid EDX image, CdSe dot is approximately at the center of the rod, consistent with the schematic structure of the CdSe@CdS DIR in (f).
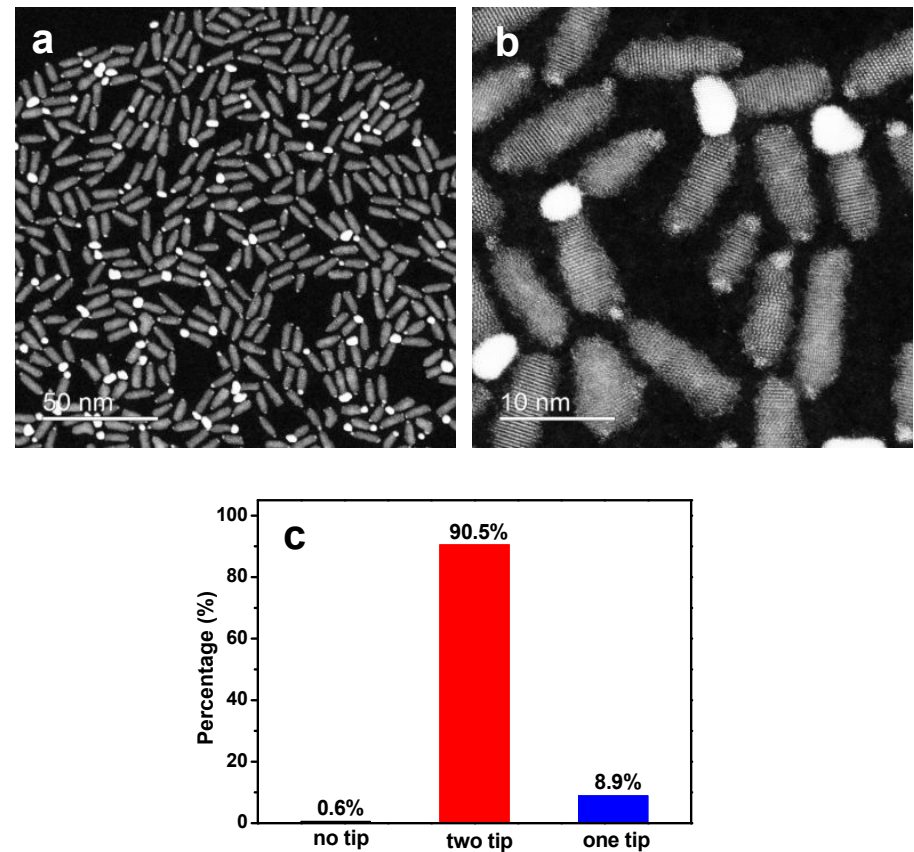

Figure S4. (a) A representative HAADF-STEM image and (b) a high resolution 
image of CdSe@CdS DIR-Pt heterostructures. (c) Histogram of percentage of DIRs with none, one, or two Pt tips.

a
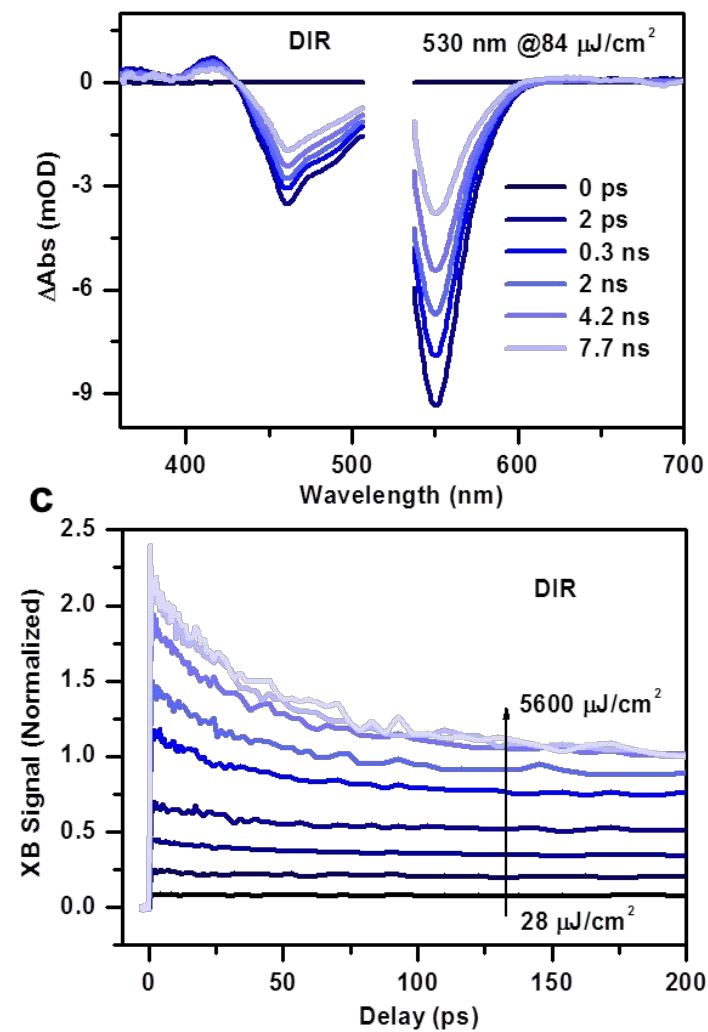

b
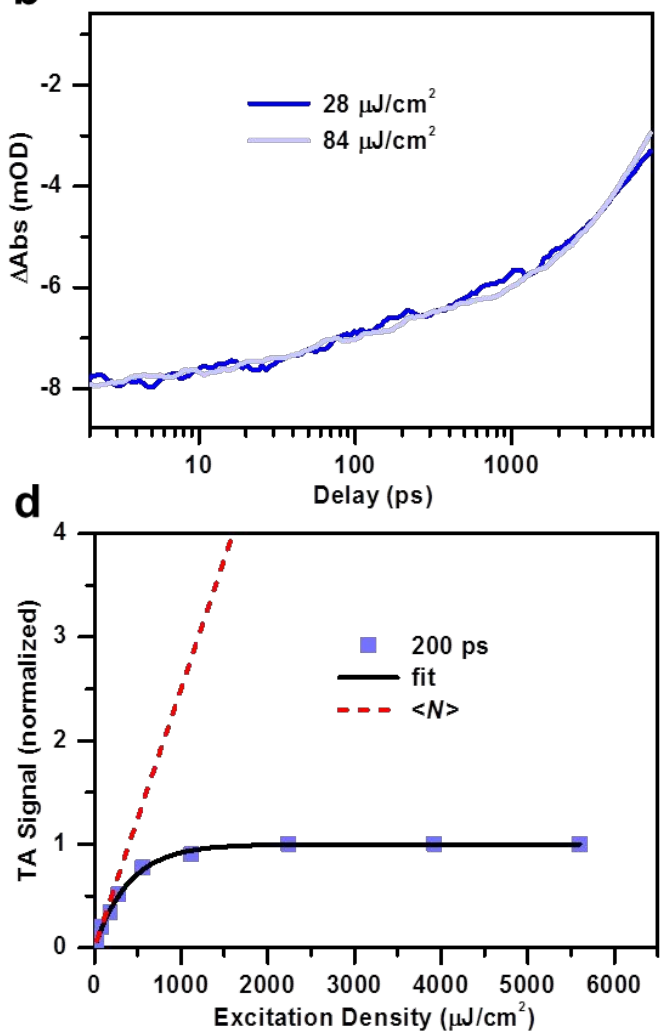

Figure S5. (a) Transient absorption (TA) spectra of CdSe@CdS DIRs probed at indicated time delays (blue lines) following $520 \mathrm{~nm}$ excitation which selectively excites CdSe core and creates $\sim 0.21$ excitons per CdSe QD $(<N>\sim 0.21)$. We use the lowest exciton bleach feature $\sim 550 \mathrm{~nm}$ (B1) to probe carrier dynamics. (b) Representative TA kinetics probed at the B1 for DIRs excited with pump fluences of 28 and $84 \mu \mathrm{J} / \mathrm{cm}^{2}$. (c) TA kinetics probed at the B1 for DIR excited with various pump fluences from 28 to $5600 \mu \mathrm{J} / \mathrm{cm}^{2}$ (blue lines); they are rescaled by normalizing the saturated long-lived component to $\sim 1$. (d) Normalized B1 signal at a delay time of 200 ps, when Auger recombination has finished, measured as a function of the excitation fluence (blue squares) and its fit to a Poisson statistics model (black solid line). According to this model, the photon absorption events when using above band-gap excitation follows a Poisson distribution, and hence, the XB signal at a delay time of $200 \mathrm{ps}$ is proportional to the fraction of excited DIRs, which can be 
expressed as: $I \propto 1-P(0)=1-e^{-<N>}$, where $<N>$ is the average number of photons absorbed per CdSe QD. $\langle N>$ is proportional to the pump laser fluence: $\langle N\rangle=C j$. Therefore, the $\mathrm{XB}$ signal at 200 ps can be fitted with: $\mathrm{I} \propto 1-e^{-C j}$, with $\mathrm{C}$ as the only fitting parameter. Using the fitted $\mathrm{C}$ value $(0.0025 \pm 0.0001)$, the average exciton number $\langle N\rangle$ at each pump fluence can be calculated (red dashed line).

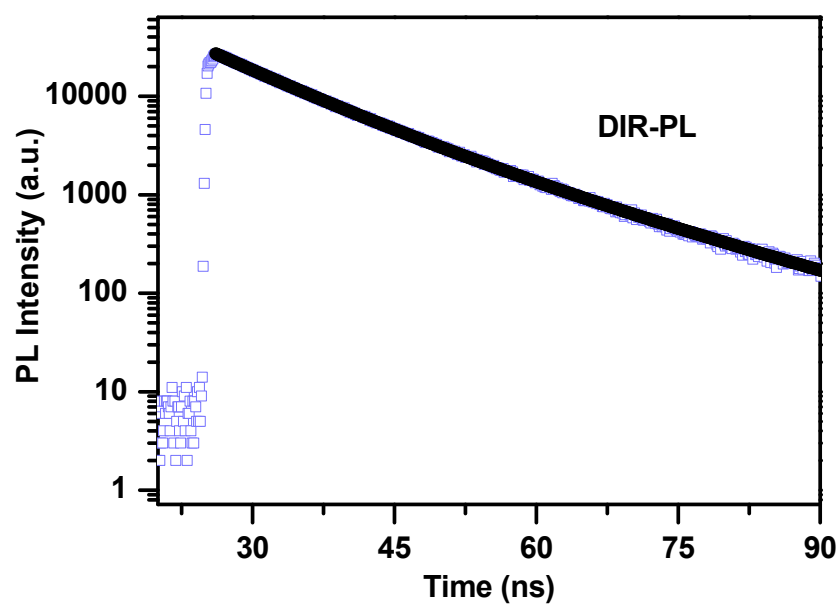

Figure S6. Time-resolved PL decay traces for CdSe@CdS DIRs (blue squares) measured under $520 \mathrm{~nm}$ excitation and its fit (black solid line). The decay for DIRs is near single-exponential with a time constant of $10.8 \pm 0.05 \mathrm{~ns}$.

a

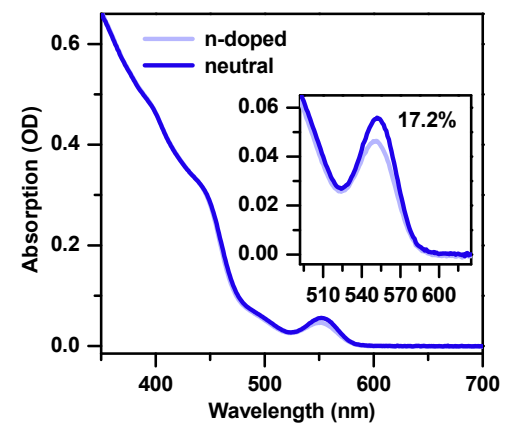

b

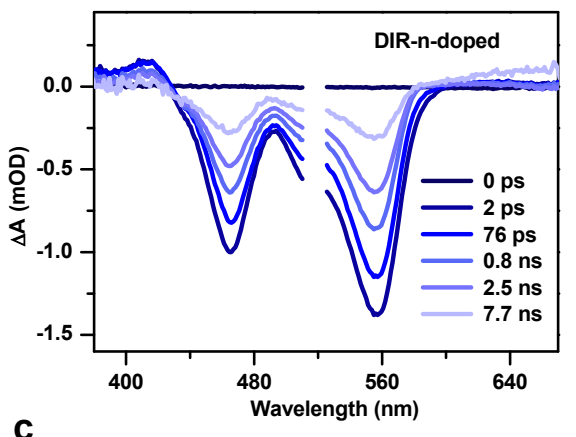

C

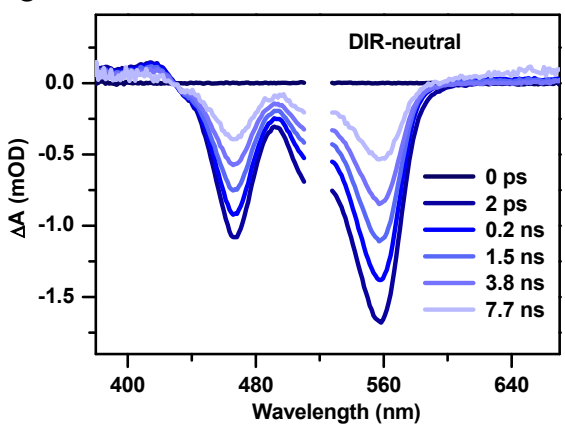


Figure S7. (a) Absorption spectra of n-doped (light blue) and neutral (blue) DIRs with the same concentration. TA spectra of (b) n-doped and (c) neutral CdSe@CdS DIRs probed at indicated delays following the excitation by a 520 pump pulse. The neutral sample was prepared by exposing the n-doped sample to the air, which released the doped electrons in a few seconds. Although the TA spectral shapes of n-doped and neutral samples are similar, the signal amplitude is lower in the former because the bleaching effect of the doped electron lowered the absorption of the sample at the pump wavelength as compared to the neutral sample, and the signal decays in the former because of Auger recombination of the negative trion.

a

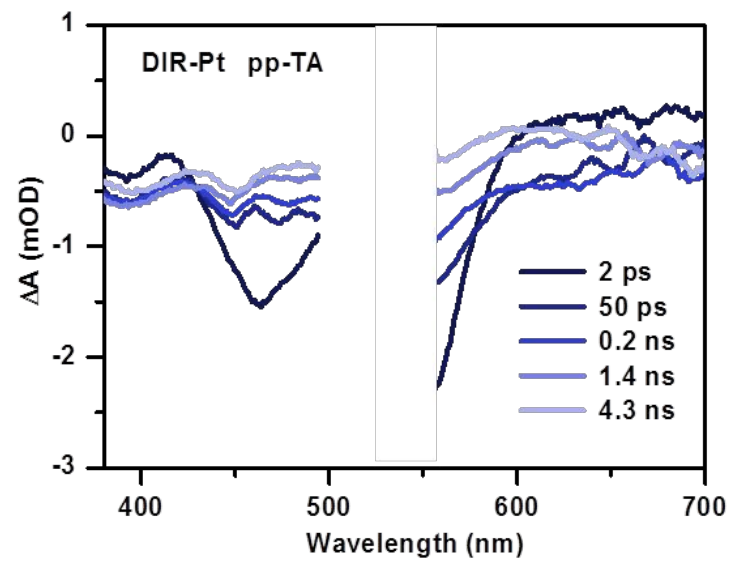

b

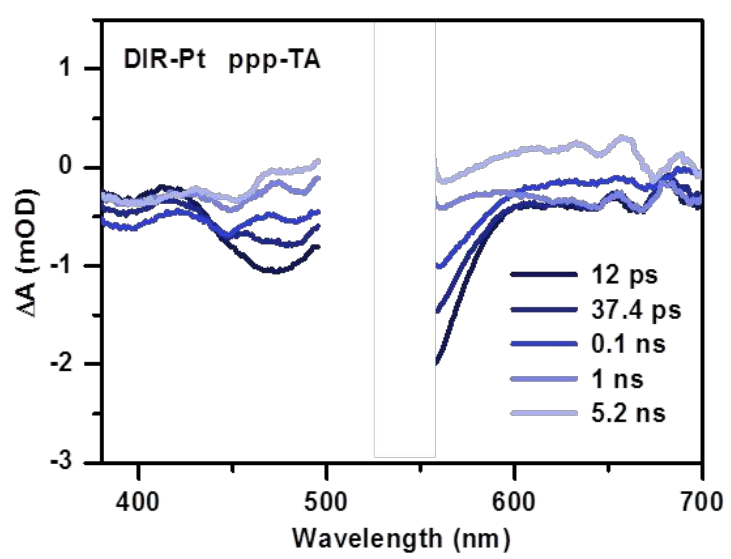

Figure S8. TA spectra of DIR-Pt probed at indicated delays following the excitation by a 520 pump pulse under (a) pp-TA and (b) ppp-TA.

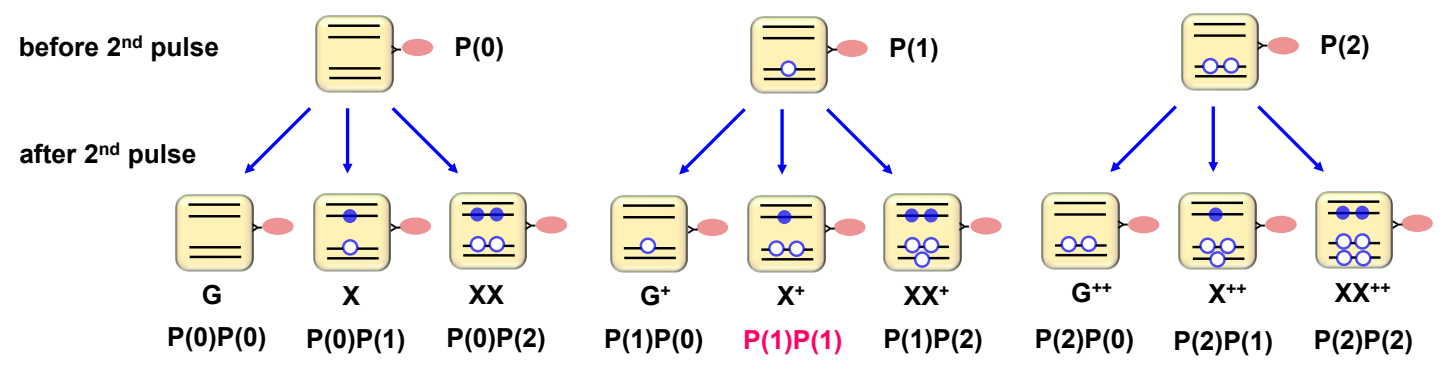

Figure S9. Analyzing the probabilities of various species in ppp-TA experiments. 


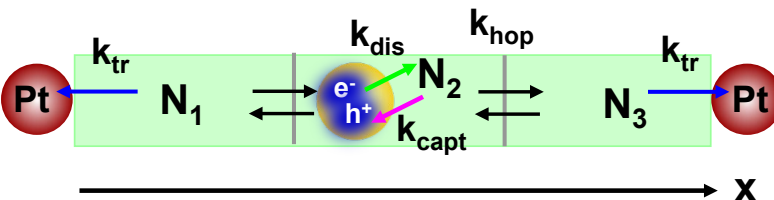

Figure S10. Model for dissociation-limited long-range electron transfer from the dot to the tip in DIR-Pt. The green arrow represents dissociation of the electron from the CdSe dot with a rate constant of $k_{\text {dis }}$, the pink arrow represents recapturing of the "free" electron back to the CdSe dot with a rate constant of $k_{\text {capt, }}$ the black arrow indicates electron hopping along the CdS rod via random walk with a rate constant of $k_{\text {hop, }}$, and the blue arrow indicates electron trapping to the Pt tip with a rate constant of $k_{\text {tr. }}$ On the basis of the electron scattering length (or mean free path) of $\sim 4 \mathrm{~nm}$ calculated for $\mathrm{CdS}$, the $\mathrm{CdS}$ rod with a length of $12.5 \mathrm{~nm}$ is divided into three segments with each segment representing a mean free path.
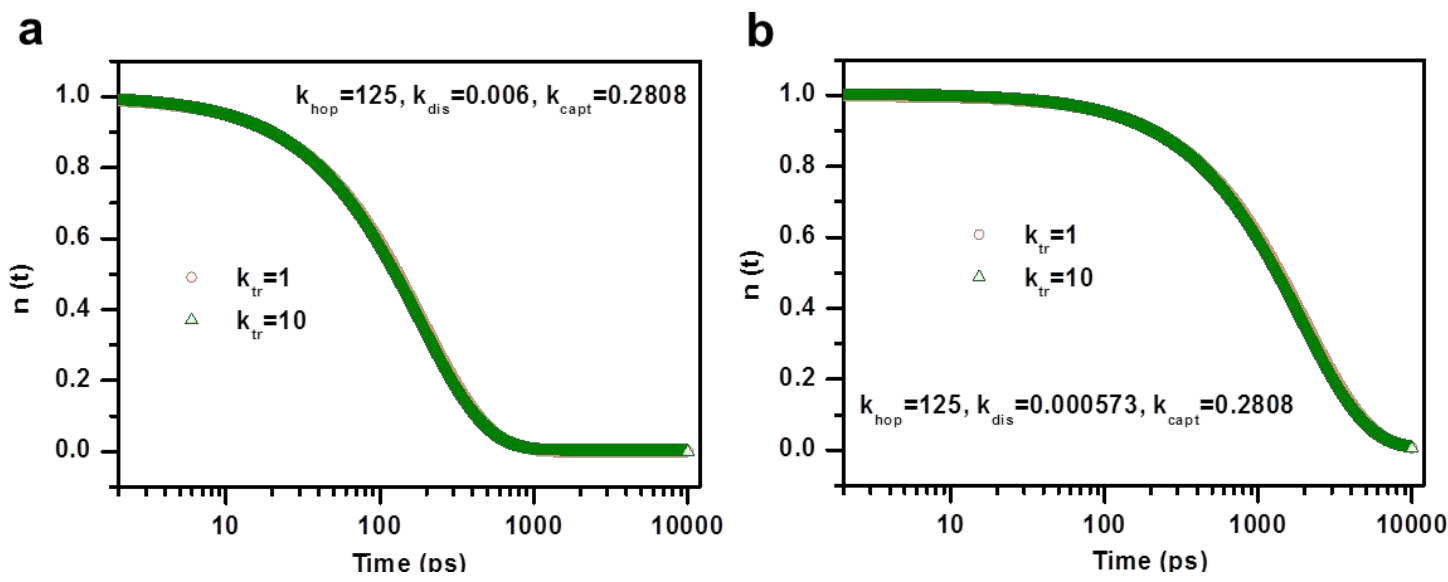

Figure S11. The probability density of electrons on CdSe dots as a function of time when $k_{\text {tr }}$ changes from 1 to $10 \mathrm{ps}^{-1}$ for (a) ET-1 and (b) ET-2. All the rate constants have a unit of $\mathrm{ps}^{-1}$. 


\section{Sample preparations}

Synthesis of CdSe@CdS DIRs. CdSe@CdS DIRs were synthesized using literature methods. ${ }^{1}$ Wurtzite CdSe QDs with $2.4 \mathrm{~nm}$ in diameter were used as seeds for the epitaxy of CdS rods. Briefly, for synthesizing CdSe@CdS DIRs, 0.06 g cadmium oxide (CdO), 3 g tri-n-octylphosphine oxide (TOPO), 0.29 g octadecylphosphonic acid (ODPA), and $0.08 \mathrm{~g}$ hexylphosphonic acid (HPA) were degassed under vacuum for 1 hour at $150{ }^{\circ} \mathrm{C}$. After heating to $350{ }^{\circ} \mathrm{C}$ under argon for half an hour, the mixture turned into clear solution, indicating the dissolution of $\mathrm{CdO}$. At this point, $1.8 \mathrm{~mL}$ TOP was injected into the solution. In a separated container, sulfur injection solution (0.12 g S in $1.8 \mathrm{~mL}$ TOP) was mixed with CdSe QDs. The amount of seeds in a typical synthesis was $8 \times 10^{-8} \mathrm{~mol}$. When the temperature of the Cd-containing solution was stabilized at $350{ }^{\circ} \mathrm{C}$, the seed-containing Sulfur injection solution was quickly injected. The growth time of CdSe@CdS DIRs was set to $1 \mathrm{~min}$. The products were precipitated out of the reaction crudes by addition of ethanol. The precipitation processes were repeated for several times. Final DIRs were dispersed in toluene.

Preparation of DIR-Pt heterostructures. CdSe@CdS NRs with Pt tips were synthesized according to a reported procedure with slight modifications. ${ }^{2,3}$ Briefly, a mixture of $0.2 \mathrm{~mL}$ oleic acid, $0.2 \mathrm{~mL}$ oleylamine, $43 \mathrm{mg}$ 1,2-hexadecanediol and 10 $\mathrm{mL}$ diphenyl ether was degassed under vacuum for $1 \mathrm{~h}$ at $80{ }^{\circ} \mathrm{C}$. The temperature of the solution was then raised to $205{ }^{\circ} \mathrm{C}$ under argon. $6 \mathrm{mg} \mathrm{Pt}$ acetylacetonate was added to a suspension of NRs in dichlorobenzene and sonicated for 5 minutes to dissolve the Pt precursor. This solution was injected into the diphenyl ether solution. The reaction was allowed to proceed for $\sim 7 \mathrm{~min}$ and quenched by water bath. The products were washed once by precipitation with ethanol followed by centrifugation, and finally dispersed in toluene.

Photochemical $n$-doping of CdSe@CdS DIRs. Photochemical doping experiments followed reported procedures. ${ }^{4}$ Briefly, $\mathrm{LiEt}_{3} \mathrm{BH}$ (1 $\mathrm{M}$ solution in tetrahydrofuran) was diluted to $0.01 \mathrm{M}$ with toluene. The CdSe@CdS DIRs solution had an optical density of $\sim 0.056$ at the band-edge peak. This solution was transferred into a glove box with a $\mathrm{N}_{2}$ atmosphere (oxygen level $<0.1 \mathrm{ppm}$ ). The diluted $\mathrm{LiEt}_{3} \mathrm{BH}$ solution 
was added into the DIRs-toluene solution under vigorous stirring and under room light. The solution was then illuminated by a UV lamp to accelerate the reaction until it reached an equilibrium. For successful photochemical doping, 10-to-100 equivalents of $\mathrm{LiEt}_{3} \mathrm{BH}$ per DIR was required, consistent with previous reports. ${ }^{5}$ The degree of doping could be controlled by varying the amount of $\mathrm{LiEt}_{3} \mathrm{BH}$ used in the reaction. The doped DIRs solution was sealed in a custom-made airtight cuvette (optical path $1 \mathrm{~mm}$ ) and transferred out of the glove box for all optical measurements. The neutralized solution was obtained by exposing the doped DIRs solution to the air for a few minutes.

\section{TA experiment set-ups}

Pump-probe experiment. The femtosecond pump-probe TA measurements were performed using a regenerative amplified Ti:sapphire laser system (Coherent; $800 \mathrm{~nm}$, $70 \mathrm{fs}, 6 \mathrm{~mJ} /$ pulse, and $1 \mathrm{kHz}$ repetition rate) as the laser source. Details were described elsewhere. ${ }^{6,7}$

Pump-pump-probe experiment. The pump-pump-probe experiments were performed by adding another pump pulse into the regular TA set-ups. Specifically, the $520 \mathrm{~nm}$ pump beam used above was split into two beams. Time delay between the pulses in these two pump beams was created by delaying one of them using a longer path length. In our experiment, this delay was fixed at 2 ns. The delay between the second pump pulse and the probe pulse was controlled by a motorized delay stage. These two beams were overlapped with the probe beam on the sample. The first pump beam is always on while the second one is chopped at $500 \mathrm{~Hz}$. The intensities of both pump beams were controlled by neutral-density filters.

\section{Calculation of trion lifetimes}

This calculation was based on previously-established statistical scaling laws for radiative and Auger nonradiative recombination rates of multi-carrier states. ${ }^{8}$ The radiative lifetime of biexcitons $\left(\tau_{X X, r}\right)$ is related to the radiative lifetime of single 
excitons $\left(\tau_{X}\right)$ via:

$$
\tau_{X X, r}=\tau_{X} / 4
$$

The radiative lifetime of trions, positive or negative, $\left(\tau_{X+(-), r}\right)$ is related to $\tau_{X}$ via:

$$
\tau_{X+(-), r}=\tau_{X} / 2
$$

The lifetime of biexcitons $\left(\tau_{X X}\right)$ is contributed by its radiative $\left(\tau_{X X, r}\right)$ and Auger $\left(\tau_{X X, A}\right)$ lifetimes via:

$$
1 / \tau_{X X}=1 / \tau_{X X, r}+1 / \tau_{X X, A}
$$

This expression also holds for positive and negative trions. The Auger lifetimes of biexcitons and trions follow a superposition relationship:

$$
1 / \tau_{X X, A}=2 / \tau_{X+, A}+2 / \tau_{X-, A}
$$

Using Eqs. S1-S4, all the lifetimes for biexcitons and trions can be calculated, which are summarized in Table S1.

Table S1. Lifetimes of multi-carrier states in CdSe@CdS DIRs.

\begin{tabular}{|l|l|l|l|}
\hline & \multicolumn{1}{|c|}{$\tau(\mathrm{ns})$} & \multicolumn{1}{c|}{$\tau_{r}(\mathrm{~ns})$} & \multicolumn{1}{c|}{$\tau_{A}(\mathrm{~ns})$} \\
\hline $\mathrm{X}$ & $10.8 \pm 0.05$ & $10.8 \pm 0.05$ & $\mathrm{NA}$ \\
\hline $\mathrm{X}^{+}$ & $0.114 \pm 0.005$ & $5.4 \pm 0.03$ & $0.116 \pm 0.005$ \\
\hline $\mathrm{X}^{-}$ & $0.826 \pm 0.044$ & $5.4 \pm 0.03$ & $0.975 \pm 0.063$ \\
\hline $\mathrm{XX}$ & $0.051 \pm 0.002$ & $2.7 \pm 0.01$ & $0.052 \pm 0.002$ \\
\hline
\end{tabular}

\section{Population analysis in ppp-TA experiments}

The first row in Fig. S8 shows the species generated by the first pump pulse before the arrival of the second pulse. From left to right are unexcited CdSe dots, dots excited with one exciton (with one hole remaining after electron transfer), and dots excited with two excitons, with probabilities of $\mathrm{P}(0), \mathrm{P}(1)$, and $\mathrm{P}(2)$, respectively. 
According to a Poisson distribution, for $\langle N>=0.1, \mathrm{P}(0)=0.905, \mathrm{P}(1)=0.09$, and $\mathrm{P}(2)$ $=0.011$, which accounts for almost all the possible species already. The second row shows the species created by the second pulse on the basis of the first pulse; their probabilities are indicated. The calculated probabilities for states $\mathrm{G}, \mathrm{X}, \mathrm{XX}, \mathrm{G}^{+}$, and $\mathrm{X}^{+}$are $0.819,0.081,0.010,0.081$, and 0.008 , which in total accounts for $99.9 \%$ of the total species.

In the ppp-TA experiments, only those species resulting from the second pulse excitation will be reflected as a difference signal. Thus, for $\langle N\rangle=0.1$, the signal can be expressed as: $S(\mathrm{ppp})=0.081 S(\mathrm{X})+0.010 S(\mathrm{XX})+0.008 S\left(\mathrm{X}^{+}\right)$. Similarly, in the regular pp-TA experiments, the signal can be expressed as: $S(\mathrm{pp})=0.09 S(\mathrm{X})+$ $0.011 S(\mathrm{XX})$. Although biexciton signal $S(\mathrm{XX})$ is almost half trion signal $S\left(\mathrm{X}^{+}\right)$in the ppp-TA, a subtraction between $S(\mathrm{ppp})$ and $S(\mathrm{pp})$ after proper scaling almost simultaneously removes the contributions of $S(\mathrm{X})$ and $S(\mathrm{XX})$ because of their similar ratio $(\sim 8: 1)$ in both type of measurements.

For $\langle N\rangle=0.2(<N>=0.3)$, the probabilities of $\mathrm{P}(0), \mathrm{P}(1)$ and $\mathrm{P}(2)$ is $0.819,0.164$ and $0.039(0.741,0.222$ and 0.080$)$, respectively. The signal for ppp- and pp-TA can be expressed as $S(\mathrm{ppp})=0.134 S(\mathrm{X})+0.032 S(\mathrm{XX})+0.027 S\left(\mathrm{X}^{+}\right)$and $\mathrm{S}(\mathrm{pp})=$ $0.164 S(\mathrm{X})+0.039 S(\mathrm{XX})$ for $\langle N>=0.2$, and $S(\mathrm{ppp})=0.165 S(\mathrm{X})+0.059 S(\mathrm{XX})+$ $0.049 S\left(\mathrm{X}^{+}\right)$and $S(\mathrm{pp})=0.222 S(\mathrm{X})+0.080 S(\mathrm{XX})$ for $\langle N\rangle=0.3$. Therefore, the contribution of $S\left(\mathrm{X}^{+}\right)$can also be satisfactorily isolated via a subtraction between $S(\mathrm{ppp})$ and $S(\mathrm{pp})$, because of the same ratio between $\mathrm{S}(\mathrm{X})$ and $\mathrm{S}(\mathrm{XX})$ in ppp- and pp-TA experiments. Therefore, the number of excitons $(<N>)$ per CdSe dot in DIR-Pt heterostructures in the ppp-TA were controlled in the range of 0.1 to 0.3 .

\section{Kinetics fitting models and electron transfer efficiency}

For DIR-Pt samples under $520 \mathrm{~nm}$ excitation, bi-exponentials are required to fit the bleach recovery kinetics:

$$
S_{X B}(t) \propto \sum_{i=1}^{2} A_{i} e^{-k_{i} t}
$$

where $A_{i}, k_{i}$ are the amplitude and rate constant of the $i$-th component, respectively. 
The fitting parameters for pp- and ppp-TA experiments are tabulated in Table S2; note that the rate constants in the above equations are all converted to time constants in the table.

The average time constant $\left(\tau_{\text {ave }}\right)$ are calculated using the following expression:

$$
\tau_{\text {ave }}=\sum_{i=1}^{2} \frac{A_{i}}{k_{i}}
$$

The time constants for ET1 and ET2 are obtained by subtracting the intrinsic decay rates of $\mathrm{X}$ and $\mathrm{X}^{+}$, respectively, from the average decay rates:

$$
\begin{aligned}
& \tau_{E T 1}=1 /\left(1 / \tau_{\text {ave }}-1 / \tau_{X}\right) \\
& \tau_{E T 2}=1 /\left(1 / \tau_{\text {ave }}-1 / \tau_{X^{+}}\right)
\end{aligned}
$$

The electron transfer efficiency $\left(\Phi_{E T}\right)$ can be calculated using the following expression:

$$
\Phi_{E T}=\tau_{\text {ave }} / \tau_{E T}
$$

Table S2. Fitting parameters for ET1 and ET2 kinetics.

\begin{tabular}{|c|c|c|c|c|c|}
\hline & $\tau_{1}\left(\mathrm{ps}, \mathrm{A}_{1}\right)$ & $\tau_{2}\left(\mathrm{ps}, \mathrm{A}_{2}\right)$ & $\tau_{\mathrm{ave}}(\mathrm{ps})$ & $\tau_{\mathrm{ET}}(\mathrm{ps})$ & $\Phi_{\mathrm{ET}}(\%)$ \\
\hline ET1 & $\begin{array}{c}65.3 \pm 14.4 \\
(53.2 \%)\end{array}$ & $\begin{array}{c}325 \pm 64 \\
(46.8 \%)\end{array}$ & $187 \pm 38$ & 192 & 97.4 \\
\hline ET2 & $\begin{array}{c}37.3 \pm 9.4 \\
(41.1 \%)\end{array}$ & $\begin{array}{c}156 \pm 21 \\
(58.9 \%)\end{array}$ & $107 \pm 16$ & 1740 & 6.1 \\
\hline
\end{tabular}

\section{Dissociation-limited long-range electron transfer model}

This model has been described in detail in a previous study of electron transfer in Pt-tipped nanorods and nanoplatelets. In this model, we first divide the CdS rod into three segments on the basis of its length $(12.5 \mathrm{~nm})$ and the electron scattering length (or mean free path; $L_{e}$ ) of $4 \mathrm{~nm}$ in $\mathrm{CdS}^{9}$. Thus, each segment corresponds to an electron mean free path and a "free" electron on CdS rod hops between neighboring 
segments (Fig. S10). The CdSe dot is placed at the center of the second segment.

The electron density in each rod segment is $N_{i}$ ( $i$ from 1 to 3 ) and the electron density in the CdSe dot is $n$. We further define the rates of electron dissociation from the CdSe dot, electron recapturing by the dot, electron hopping along the $\mathrm{CdS}$ rod and electron trapping into the Pt tip as $k_{\text {dis }}, k_{\text {capt }}, k_{\text {hop }}$ and $k_{\text {tr }}$, respectively (Fig. S10). With these definitions, the coupled rate equations for electron densities are:

$$
\begin{gathered}
\frac{d N_{1}(t)}{d t}=k_{\text {hop }}\left[N_{2}(t)-N_{1}(t)\right]-k_{t r} N_{1}(t) \\
\frac{d N_{2}(t)}{d t}=k_{\text {hop }}\left[N_{1}(t)+N_{3}(t)-2 N_{2}(t)\right]+k_{d i s} n(t)-k_{\text {capt }} N_{2}(t) \\
\frac{d N_{3}(t)}{d t}=k_{\text {hop }}\left[N_{2}(t)-N_{3}(t)\right]-k_{t r} N_{3}(t) \\
\frac{d n(t)}{d t}=k_{\text {capt }} N_{2}(t)-k_{d i s} n(t)
\end{gathered}
$$

Because the exciton is initially created in the CdSe dot, the initial conditions are: $n(0)$ $=1$ and $N_{i}(0)=0$.

Among the rate parameters, $k_{\mathrm{hop}}$ can be calculated according to: $k_{\mathrm{hop}}=2 D_{e} / L_{e}^{2}$, where $D_{e}$ is the electron diffusion coefficient in $\operatorname{CdS}\left(\sim 10 \mathrm{~cm}^{2} \mathrm{~s}^{-1}\right)$ and $L_{e}$ is the electron mean free path in CdS $(4 \mathrm{~nm}) .{ }^{9}$ Thus, $k_{\text {hop }}$ was estimated to be $125 \mathrm{ps}^{-1}$. Because $k_{\text {dis }}$ and $k_{\text {capt }}$ are the rates of reverse processes, we assumed that they followed the following relationship: $k_{\mathrm{dis}}=k_{\text {capt }} \cdot \exp \left(-E_{b} / k_{\mathrm{B}} T\right)$, with $E_{b}$ standing for the barrier for electron dissociation from the CdSe dot. ET1 and ET2 share the same $k_{\text {capt. }}$ Previous studies showed that interfacial electron transfer from semiconductor nanocrystals to attached Pt occurred on a sub-ps timescale. ${ }^{10}$ On our simulation, we set $k_{\text {tr }}$ at $1 \mathrm{ps}^{-1}$ and left $k_{\text {capt }}$ and $E_{b}$ as adjustable parameters. Using these parameters, we numerically solved the coupled rate equations above. We are particularly interested in the electron density in the dot $(n)$ because it is proportional to the exciton bleach amplitude of CdSe dot (B1) measured experimentally.

\section{Trion binding energy}

We express the dissociation process of neutral exciton $(\mathrm{X})$ and positive trion $\left(\mathrm{X}^{+}\right)$ using the following equations: 


$$
\begin{gathered}
X=e^{-}+h^{+}-E_{b, X} \\
X^{+}=e^{-}+2 h^{+}-E_{b, X^{+}} \\
X^{+}=X+h^{+}-E_{\text {trion }}
\end{gathered}
$$

Eq. S14 represents exciton dissociation into an electron and a hole, and $E_{b, X}$ is the exciton binding energy (defined as a positive value here); Eq. S15 represents $\mathrm{X}^{+}$ dissociation into an electron and two holes, and $E_{b, X^{+}}$is the binding energy of one electron to two holes; Eq. S16 represents $\mathrm{X}^{+}$dissociation into an exciton and a hole, and $E_{\text {trion }}$ is the binding energy of a hole to an exciton which is commonly called the trion binding energy. Plugging Eqs. S14 and S15 into Eq. S16, one immediately obtains the following relationship:

$$
E_{\text {trion }}=E_{b, X^{+}}-E_{b, X}
$$

\section{References for SI:}

(1) Carbone, L.; Nobile, C.; De Giorgi, M.; Sala, F. D.; Morello, G.; Pompa, P.; Hytch, M.; Snoeck, E.; Fiore, A.; Franchini, I. R.; Nadasan, M.; Silvestre, A. F.; Chiodo, L.; Kudera, S.; Cingolani, R.; Krahne, R.; Manna, L. Synthesis and Micrometer-Scale Assembly of Colloidal CdSe/CdS Nanorods Prepared by a Seeded Growth Approach. Nano Lett. 2007, 7, 2942-2950.

(2) Habas, S. E.; Yang, P.; Mokari, T. Selective Growth of Metal and Binary Metal Tips on CdS Nanorods. J. Am. Chem. Soc. 2008, 130, 3294-3295.

(3) Wu, K.; Chen, Z.; Lv, H.; Zhu, H.; Hill, C. L.; Lian, T. Hole Removal Rate Limits Photo-driven H2 Generation Efficiency in CdS-Pt and CdSe/CdS-Pt Semiconductor Nanorod-metal tip Heterostructures. J. Am. Chem. Soc. 2014, 136, 7708-7716.

(4) Rinehart, J. D.; Schimpf, A. M.; Weaver, A. L.; Cohn, A. W.; Gamelin, D. R. Photochemical Electronic Doping of Colloidal CdSe Nanocrystals. J. Am. Chem. Soc. 2013, 135, 18782-18785.

(5) Ding, T.; Liang, G.; Wang, J.; Wu, K. Carrier-doping as a tool to probe the electronic structure and multi-carrier recombination dynamics in heterostructured colloidal nanocrystals. Chem. Sci. 2018, 9, 7253-7260.

(6) Wang, J.; Ding, T.; Wu, K. Charge Transfer from n-Doped Nanocrystals: Mimicking Intermediate Events in Multielectron Photocatalysis. J. Am. Chem. Soc. 2018, 140, 7791-7794. 
(7) Wang, J.; Ding, T.; Wu, K. Electron Transfer into Electron-Accumulated Nanocrystals: Mimicking Intermediate Events in Multielectron Photocatalysis II. $J$. Am. Chem. Soc. 2018, 140, 10117-10120.

(8) Wu, K.; Lim, J.; Klimov, V. I. Superposition Principle in Auger Recombination of Charged and Neutral Multicarrier States in Semiconductor Quantum Dots. ACS Nano 2017, 11, 8437-8447.

(9) Wu, K.; Hill, L. J.; Chen, J.; McBride, J. R.; Pavlopolous, N. G.; Richey, N. E.; Pyun, J.; Lian, T. Universal Length Dependence of Rod-to-Seed Exciton Localization Efficiency in Type I and Quasi-Type II CdSe@CdS Nanorods. ACS Nano 2015, 9, 4591-4599.

(10) Wu, K.; Li, Q.; Du, Y.; Chen, Z.; Lian, T. Ultrafast exciton quenching by energy and electron transfer in colloidal CdSe nanosheet-Pt heterostructures. Chemical Science 2015, 6, 1049-1054. 